\title{
NON-INVASIVE VIBRATING CONTROL OF THE ZOSER PYRAMID
}

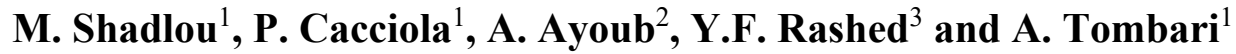 \\ ${ }^{1}$ School of Environment and Technology, University of Brighton, Brighton, UK \\ e-mail: \{m.shadlou, p.cacciola, a.tombari\}@brighton.ac.uk \\ ${ }^{2}$ School of Mathematics, Computer Science and Engineering, City University of London, UK \\ email: ashraf.ayoub.1@city.ac.uk \\ ${ }^{3}$ Department of Structural Engineering, Cairo University, Giza, Egypt, \\ email: youssef@eng.cu.edu.eg
}

\begin{abstract}
The historical sites and monuments in Egypt are among the most important in the world and conserving this cultural heritage is crucial. The large damages observed in hundreds of monuments and in the Zoser Pyramid after the Cairo Earthquake in 1992 unveiled the fragility of the Egyptian heritage asset to seismic hazards. This paper aims to investigate the use of the innovative non-invasive vibration control device, the vibrating barrier (ViBa) as a potential strategy to mitigate the effects of the seismic action on the Zoser Pyramid. In this regard, a finite element model of the Zoser Pyramid and surrounding soil has been developed. A pertinent ground motion acceleration induced by the 1992 Cairo earthquake event has been simulated through spectral representation method with power spectral density function defined by seismological data available for Saqqara. Moreover, a reduced-order model is implemented to facilitate the design of the ViBa device. The efficiency of the ViBa is assessed by numerical simulation of the finite element model of the ViBa-Soil-Pyramid system. Results show an evident reduction of the acceleration and stresses on the Pyramid, especially in those areas where the damages have been observed.
\end{abstract}

Keywords: Vibrating Barriers, Zoser Pyramid, Soil-Structure Interaction. 


\section{INTRODUCTION}

Egypt has an extensive cultural heritage, reaching back more than five thousand years, with a plethora of archaeological sites considered among the most important in the world. The archaeological site of Saqqara, located at a distance of about $20 \mathrm{~km}$ from Cairo city, is appraised as the world's most extensive burial ground with monuments of almost every period of ancient Egyptian civilization. In this area, the Step Pyramid of Djoser or Zoser's pyramid is located. It is therefore an important legacy of ancient construction to be preserved.

Although Egypt is a region of small to moderate magnitude earthquakes [1], several events that occurred in the area from the late 1980s, with two main events, the 1992 Cairo earthquake and the 1995 Gulf of Aqaba earthquake, partly damaged the Zoser pyramid that needed of an important restoration activity to avoid the risk of collapse leading to its closure to visitors for a long time [1]. Therefore, it becomes evident that the preservation and conservation of archaeological monuments and sites require unconventional and exceptional seismic analysis and design. Conventional seismic protection systems mainly based on strengthening techniques or local devices such as dampers or seismic isolators are based on structural invasive interventions whose application clearly might risk compromising the historical value of heritage structures such as the pyramid. Those techniques are generally applied to heritage structures as a repairing technique (as done to the Zoser pyramid) rather than as a preventive strategy to mitigate future seismic actions. Ancient monuments, therefore, are left generally unprotected from future catastrophic events due to the difficulty to protect them without altering their historical value.

In this paper, the novel non-invasive seismic passive control device [2] called Vibrating Barrier ( ViBa) is proposed as a mean of protection for the Zoser Pyramid. The ViBa is a device buried in the soil and detached from the surrounding structure able to absorb a significant portion of the dynamic energy arising from the ground motion. It exploits the basic principle of the structure-soil-structure interaction introduced in the early 70's [3][4]. To date, several studies on the efficiency of the ViBa have been carried out to mitigate the seismic vulnerability of different structures: Cacciola et al. [5] investigated the potential of ViBa for the seismic protection of monopiled structures, Tombari et al. [6]considered ViBa to mitigate the seismic risk of a nuclear reactor, Cacciola et al. [7] and Andreozzi et al. [8]applied ViBa to control the seismic response of heritage buildings, while the ViBa device was used to protect a large urban area in Coronado et al. [9] as well as a cluster of buildings in Tombari et al. [10]. Therefore, to mitigate the seismic response of the Zoser pyramid, the ViBa device has to be properly designed. In this regard, due to the lack of ground motion records in the area, a stochastic approach following the Boore method [11], has been adopted to determine the power spectral density function of the ground motion ad the bedrock underneath the Zoser pyramid. The model has been calibrated using the seismological parameters related to the Cairo 1992 earthquake [12]. The stochastic model has been then used as seismic input for the design of the ViBa device.

Furthermore, a sub-structuring method is proposed in the paper to reduce the large order of the complete system composed of Pyramid and soil and the ViBa. Following this approach, it is possible to optimize the $\mathrm{ViBa}$ to minimize the effect of the ground motion on selected-response parameters (e.g. displacement, stresses). Several configurations of ViBas are proposed and their impact on the seismic response of the Pyramid is computed employing a pertinent Monte Carlo Simulation. The efficiency of the ViBa is assessed in terms of mitigation of the peak acceleration of the top of the pyramid and Von Mises stresses over one of the areas that has been damaged during the 1992 Cairo Earthquake. 


\section{NUMERICAL MODELLING OF ZOSER PYRAMID}

The Zoser pyramid is modelled in this study as a stepped solid structure (Figure 1a) with a rectangular base of about $108 \mathrm{~m} \times 120 \mathrm{~m}$ and a total height of about $63 \mathrm{~m}$. Surveying with a 3D scanner has been carried on to determine the geometry of the Zoser pyramid. Soil investigations have been also conducted at the site. Specifically, samples from the stones inside the site were tested in the laboratory for determining the properties of the materials used in building the pyramid. Moreover, a borehole with a depth of 10 meters was dug to determine the soil properties in Saqqara. The total borehole was limestone or sandy limestone interspersed with layers of silt at the depth of the borehole. No groundwater has been observed during the borehole. Geometrical dimensions of the simplified geometry used in this study pertinent to North and West sections are reported in Figure $1 \mathrm{~b}$ and 1c, respectively. The Pyramid is made of polished white limestone modelled as an isotropic linear material defined by the elastic modulus, $E_{p}=45 \mathrm{GPa}$, Poisson's ratio, $v_{p}=0.25$ as well as unit density, $\rho_{p}$, of $2900 \mathrm{~kg} / \mathrm{m}^{3}$. The position of the bedrock has been determined from previous studies available in the literature [13], [14] and it is considered to be located at $35 \mathrm{~m}$ depth. The soil stratum between the bedrock and the base of the pyramid is assumed homogeneous with a shear wave velocity of $3000 \mathrm{~m} / \mathrm{sec}$ considering the site investigation undertaken in the first $10 \mathrm{~m}$. Material properties used to define the linear elastic behaviour of the soil and Pyramid are shown in Table 1.
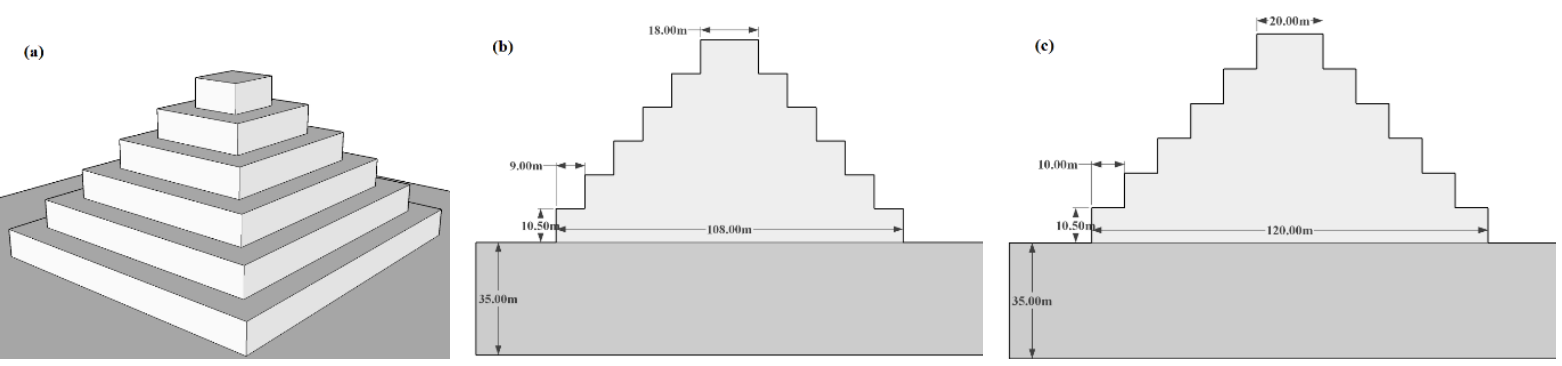

Figure 1. Zoser Pyramid, (a) 3D view, (b) North and c) West sections of the stepped Pyramid

\begin{tabular}{|ccc|ccc|}
\hline Soil & & \multicolumn{3}{c|}{ Pyramid } \\
\hline$\rho_{s}\left(\mathrm{~kg} / \mathrm{m}^{3}\right)$ & $E_{s}(\mathrm{kPa})$ & $v_{s}$ & $\rho_{p}\left(\mathrm{~kg} / \mathrm{m}^{3}\right)$ & $E_{p}(\mathrm{kPa})$ & $v_{p}$ \\
2200 & $45 \times 10^{6}$ & 0.25 & 2900 & $45 \times 10^{6}$ & 0.25 \\
\hline
\end{tabular}

Table 1. Material properties for Soil and Pyramid

Numerical Finite Element models of the fixed-base Pyramid (Figure 2a) and of the full Pyramid-Soil system (Figure 2b) are created through the software SAP2000 by using 8-node elements. A large soil model with lateral free boundaries, $500 \mathrm{~m}$ far away from each edge of the Pyramid, is considered to mitigate the spurious waves reflected back to the system. Fully fixed restraints are used on the bottom of the soil to simulate the rigid bedrock.

A modal analysis is first carried out for the fixed-base Pyramid model: the first mode, associated with its smallest width (Figure 3a) has a natural frequency of $14.6 \mathrm{~Hz}$, while the second mode, associated with the largest width (Figure $3 b$ ) is characterized by the natural frequency of $14.95 \mathrm{~Hz}$. 

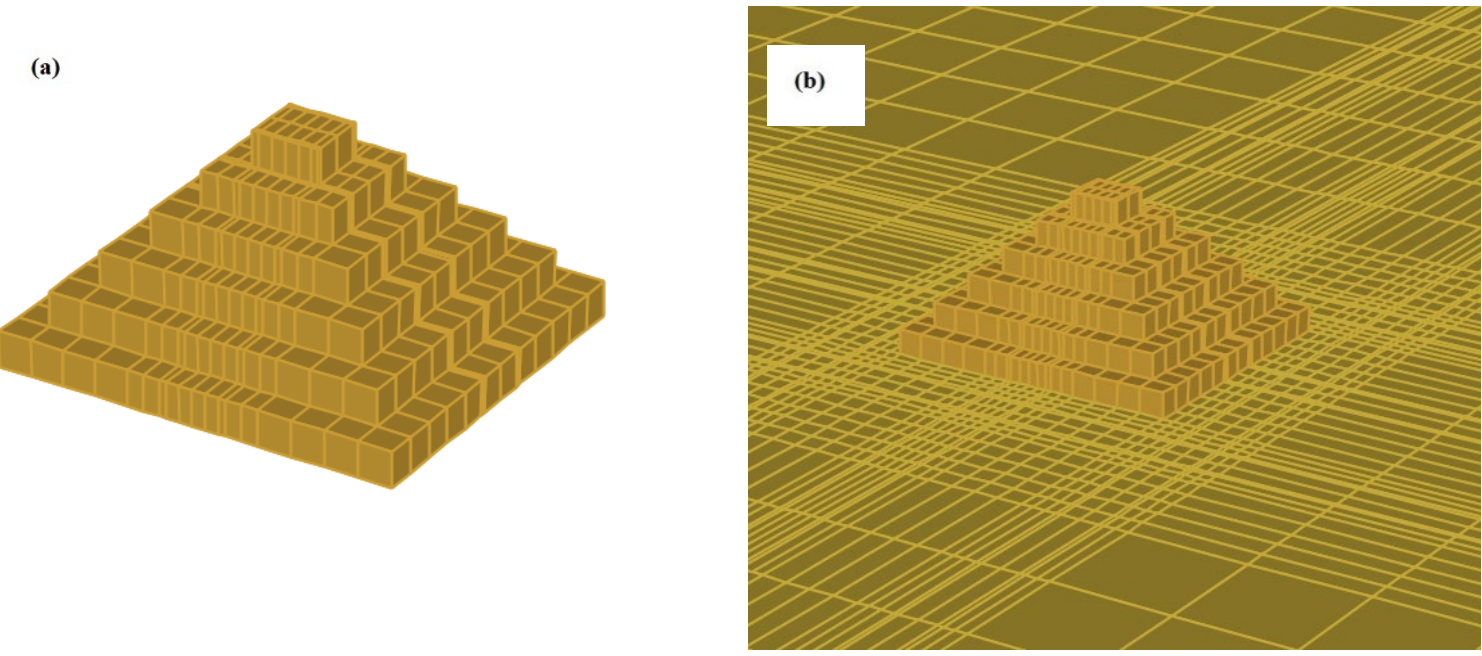

Figure 2. a) Mesh configuration of the fixed-base pyramid and b) soil-pyramid system

(a)

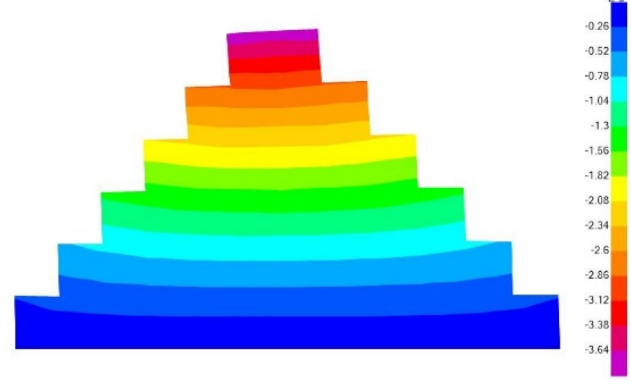

(b)

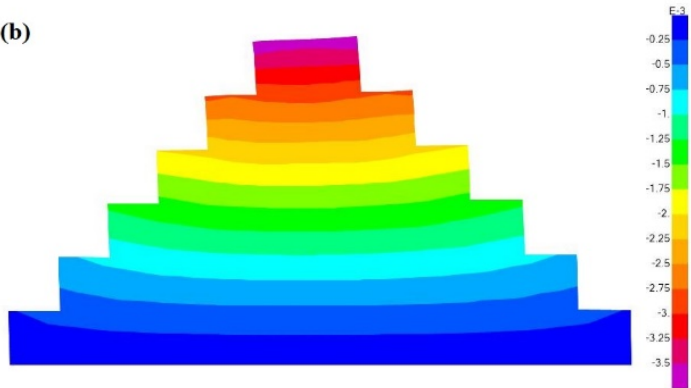

Figure 3. The amplitude of the modal vector for a) the first and b) the second mode of the Pyramid.

Successively, the modal analysis is carried out on the full Soil-Pyramid system to account for the soil-structure interaction effects: the first natural frequency of the pyramid is shifted from $14.6 \mathrm{~Hz}$ to $10.54 \mathrm{~Hz}$, while the second natural frequency changed from 14.95 to 10.73 because of the soil flexibility (Figure $4 \mathrm{a}$ and $\mathrm{b}$ ).
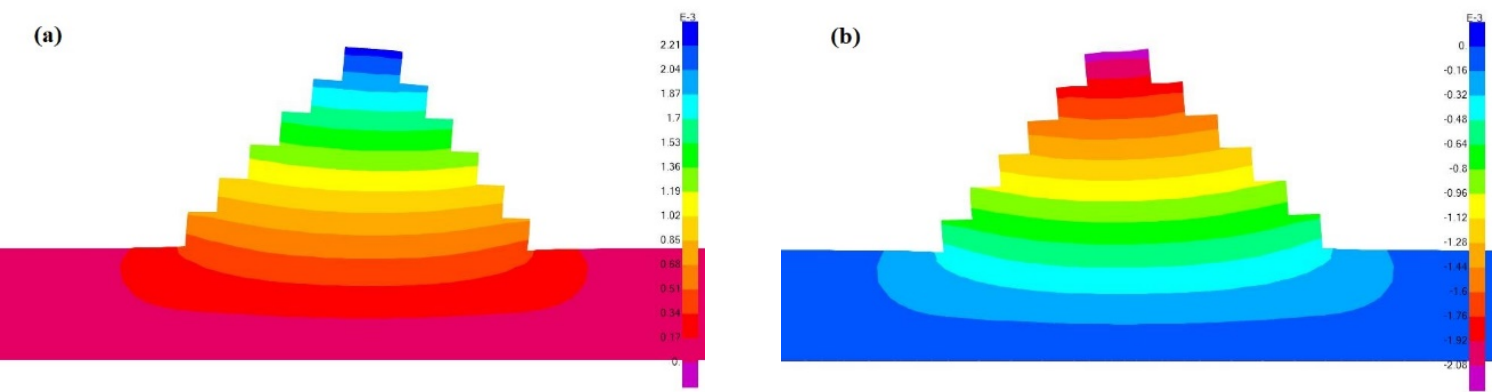

Figure 4. The amplitude of the modal vector for a) the first and b) the second mode of the soil-Pyramid system. 


\section{PROPOSED METHOD FOR THE DESIGN OF THE VIBRATING BARRIERS}

To mitigate the seismic response of the Zoser Pyramid, the non-localised system denoted Vibrating Barrier is employed in this section. Figure 5 shows a potential implementation of the Vibrating Barrier to protect the Zoser pyramid.

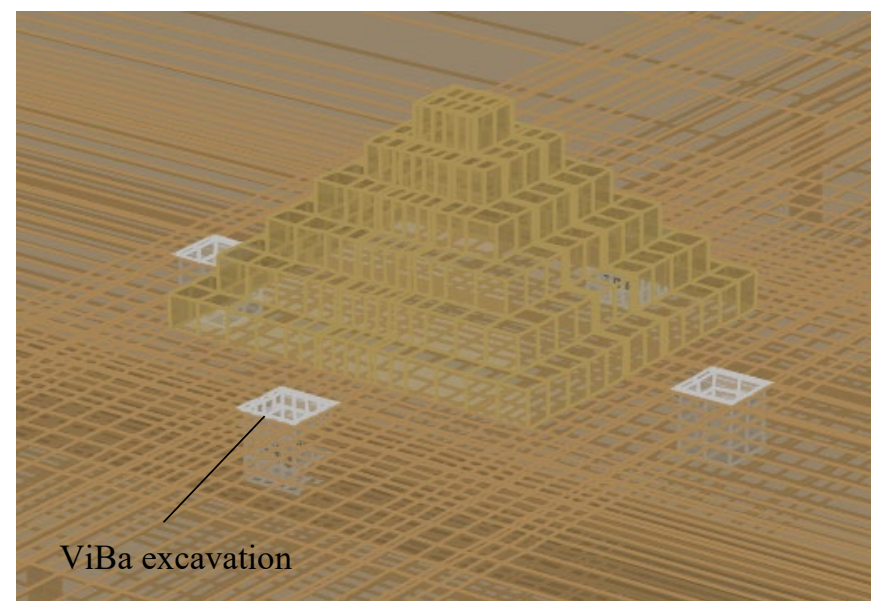

Figure 5. A potential configuration of the Vibrating Barriers to protect the Zoser pyramid

For a selected configuration, the problem to design the $\mathrm{ViBa}$ is therefore reduced to determine the mechanical parameters of the devices so that relevant response quantities (e.g., stresses, accelerations) are reduced.

A reduced-order model capturing the $\mathrm{ViBa}(\mathrm{s})$-Soil-Pyramid interaction is developed to reduce the computational complexity of the optimization problem used to design the ViBa mechanical parameters. Specifically, a sub-structuring method [15], determined in the frequency domain, is used to condensate the Soil-Pyramid system into complex-valued transfer functions and, to reduce the order of the full system to just 2 translational degrees of freedom related to the translational $\mathrm{ViBa}$ displacement, $X_{v}$ and to the displacement, $X_{f}$, of its box foundation.

Although the design method proposed in the next section is developed by considering one $\mathrm{ViBa}$ device, the approach can be easily extended for any number of ViBas and any possible configuration and location, by adopting the superposition principle.

\subsection{A Multi-Step Approach to Evaluate the ViBa-Soil-Structure Interaction}

The proposed method consists to subdivide the full domain into 2 partitions: the first subdomain represents the Soil-Pyramid system with any rigid excavations where the ViBas will be installed (Figure 6a); the second subdomain comprises the internal structure of the ViBa contained by the rigid box-foundation resting on a flexible soil medium modelled through soilfoundation impedance functions (Figure 6b). In the design approach proposed in this paper, only the translational degrees of freedom of the ViBa and its foundation, are considered. A 4step analysis is devised as follows: i) a steady-state analysis of the first subdomain subjected to bedrock ground motion displacement, $U_{b}(\omega)$, modelled as unitary constant function to derive the foundation input motion of the rigid excavation, $U_{F I M}^{V}(\omega)$, as well as the normalized displacement in a relevant point of the Pyramid, $U_{p}^{(1)}(\omega)$; ii) a steady-state analysis of the first subdomain where a unitary displacement, $X_{0}(\omega)$, is applied to the centre of the rigidity of the excavation (Figure 7) to derive the displacement in a relevant point of the Pyramid, $U_{p}^{(2)}(\omega)$, as well as the complex-valued soil-foundation impedance, $\widetilde{K}_{h}(\omega)$; iii) an inertial analysis of the ViBa-Soil subdomain where the soil is modelled through the impedance $\widetilde{K}_{h}(\omega)$; and iv) 
recovery of the complete displacement of the relevant point of the Pyramid by adopting the superposition principle. It is worth noting the control parameter of the Pyramid can be represented not just in terms of displacements but even stresses, strains, internal forces, or any relevant measure, without compromising the generality of the presented method.
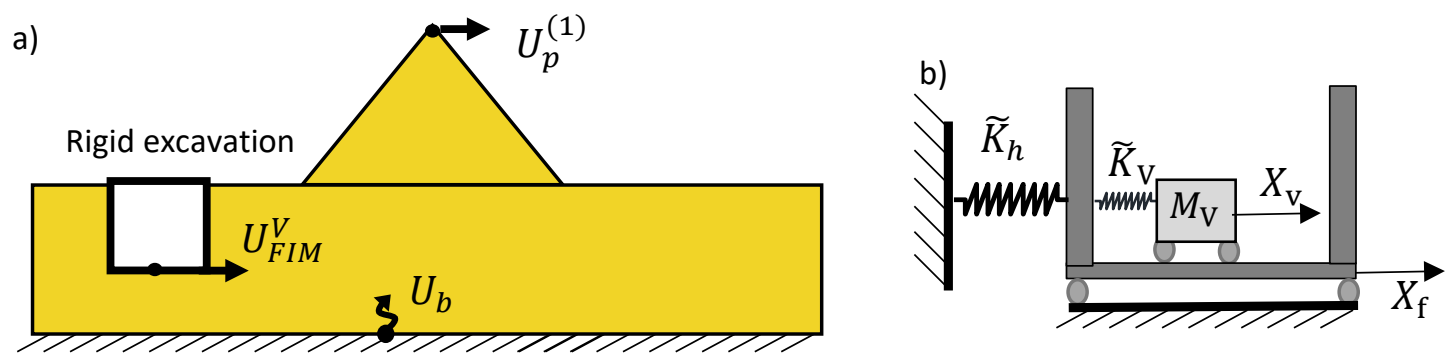

Figure 6. Sub-structuring method: a) Soil-Pyramid System; and b) ViBa-Soil System

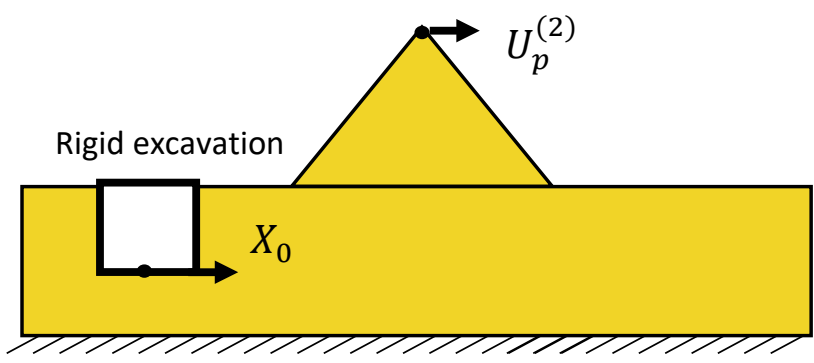

Figure 7. Steady-state analysis to condensate the Soil-Pyramid system into transfer functions

\subsection{Reduced-Order ViBa-Soil-Pyramid Model Governing Equations}

After computing the various complex-valued transfer functions, $U_{p}^{(1)}(\omega)$ in step i) and $U_{p}^{(2)}(\omega)$ in step ii), the design of the optimal ViBa parameters is performed on the reducedorder model of step iii) and iv) in which an extremely fast optimization analysis can be implemented. Considering the mechanical system of Figure $6 \mathrm{~b}$, the governing equations of the reduced-order model can be written in the frequency domain as follows:

$$
\left\{\begin{array}{l}
\widetilde{K}_{V} X_{V}-\widetilde{K}_{V} X_{\mathrm{f}}-\omega^{2} M_{V} X_{V}=0 \\
\left(\widetilde{K}_{h}+\widetilde{K}_{V}\right) X_{\mathrm{f}}-\widetilde{K}_{V} X_{V}-\omega^{2} M_{0} X_{\mathrm{f}}=\widetilde{K}_{h} U_{F I M}^{V}
\end{array}\right.
$$

where the dependencies from the frequency $\omega$ have been omitted. Eq. (1) represents a system of algebraic equations in which $X_{V}$ and $X_{\mathrm{f}}$, are the unknown ViBa's displacement and foundation displacement, respectively, whilst $\widetilde{K}_{V}$ is the ViBa parameter to be optimized. To perform the optimal design of the $\mathrm{ViBa}$, the complete displacement of the relevant point of the Pyramid, $U_{p}$, must be computed by superposition in step iv), as follows:

$$
U_{p}(\omega)=\left(U_{p}^{(1)}(\omega)+\frac{U_{p}^{(2)}(\omega)}{X_{0}(\omega)} X_{\mathrm{f}}(\omega)\right) U_{g}(\omega)
$$

where $U_{g}(\omega)$ is the Fourier transform of the earthquake ground motion displacement applied at the bedrock. It is worth mentioning that in Eq. (2), the terms within the brackets, $H(\omega)=$ 
$\left(U_{p}^{(1)}(\omega)+U_{p}^{(2)}(\omega) X_{\mathrm{f}}(\omega) / X_{0}(\omega)\right)$ represents the non-dimensional transfer function $H(\omega)=$ $\frac{U_{p}}{U_{g}}$ between the response output of the Pyramid and the input ground motion at the bedrock. Because of the derivation rule in the frequency domain, $H(\omega)=\frac{U_{p}}{U_{g}}=\frac{\dot{U}_{p}}{\dot{U}_{g}}=\frac{\ddot{U}_{p}}{\ddot{U}_{g}}$.

\subsection{Optimal design of the ViBa}

The design of the ViBa, once the mass, $M_{V}$, is assigned, involves the determination of the optimal value of the internal device, $\widetilde{K}_{V}$, which can be expressed as $\widetilde{K}_{V}=K_{V}\left(1+i \eta_{V}\right)$ with $i$ as an imaginary unit, $K_{V}$, real-valued stiffness of the internal device and, $\eta_{V}$ as its loss factor.

Therefore, Eq. (2) can be re-written as

$$
U_{p}(\omega, \alpha)=H(\omega, \alpha) U_{g}(\omega)
$$

in which the parameters to be optimised, listed in the design vector $\alpha=\left[K_{V}, \eta_{V}\right]$, are made explicit in the transfer function. The optimization approach adopted in this paper involves the mitigation of the stochastic response of the Pyramid. Considering a ground motion acceleration modelled as Gaussian zero-mean stochastic process with zero mean, fully defined by the knowledge of the power spectral density function $\operatorname{PSD}(\omega)$, it becomes straightforward to extend Eq. (3) by using the Random Vibration Theory, as follows:

$$
G(\omega, \alpha)=H^{*}(\omega, \alpha) P S D(\omega) H(\omega, \alpha)
$$

where $G(\omega, \alpha)$ is the PSD function in terms of acceleration at the reference point of the Pyramid and.$^{*}$ is the complex conjugate operator.

The largest value of the response in terms of acceleration, $\ddot{u}_{p, V i B a}^{\max }$, of the reference point of the Pyramid protected by the ViBa technology, can be derived from Eq. (4) in approximated fashion [8] as:

$$
\ddot{u}_{p, V i B a}^{\max }(\alpha) \cong \eta_{p} \sqrt{\int_{0}^{\infty} G(\omega, \alpha) d \omega}=\eta_{p} \sqrt{\lambda_{0}(\alpha)}
$$

where $\lambda_{0}$ is the zeroth-order spectral moment and $\eta_{p}$ is the peak factor.

The objective function of the optimization approach determined on the Reduced Order Model (ROM) is defined as follows:

$$
\min _{\alpha>0}\left(\ddot{u}_{p, V i B a}^{\max }(\alpha)\right)
$$

Clearly, other response parameters can be used such as the maximum stress in a specific element or the top displacement. The efficiency of the ViBa device is then measured through the reduction factor in terms of acceleration, $R_{\ddot{u}_{p}}^{R O M}$, as follows:

$$
R_{\ddot{u}_{p}}^{\text {ROM }}(\alpha)=\frac{\left|\ddot{u}_{p}^{\max }-\ddot{u}_{p, V i B a}^{\max }(\alpha)\right|}{\ddot{u}_{p}^{\max }(\alpha)} \times 100
$$

Therefore, the optimization problem can be written as minimization of the objective function which can be solved with standard iterative algorithms for constrained optimization, such as the derivative-free method [16]. 


\section{NUMERICAL RESULTS}

In this study, the method proposed in Section 3 is applied to mitigate the seismic response of the Zoser Pyramid. Three ViBa configurations (hereinafter called, Case 1-2-3), oriented in the North-South (N-S) direction as depicted in Figure 8, are proposed.

Case 1 is represented by one ViBa on the Southern side of the Pyramid realized in a rigid excavation of size $20 \mathrm{~m} \times 20 \mathrm{~m} \times 20 \mathrm{~m}$; in Case 2, one ViBa for each side of the Pyramid is considered; Case 3 considers one ViBa for each side of the Pyramid as in Case 2 but placed inside a larger rigid excavation of $60 \mathrm{~m}$ long, 20 wide and $20 \mathrm{~m}$ deep. Furthermore, in Case 3, each box-foundation contains three internal vibrating masses supposed to oscillate synchronously.

In every configuration, the excavations where the ViBas are located, are realized at a minimum distance of $15 \mathrm{~m}$ from each side of the Pyramid. The box foundation of the ViBa is made of four concrete retaining walls and a matt foundation. The reference point used for the optimization procedure of Section 3, indicated by point ' $p$ ' in Figure 8, is selected at the top of the Pyramid. Moreover, an area of interest is to calculate the stresses induced by the seismic event, denoted as representative element (RE) in Figure 8. Because of the damages that the Zoser Pyramid experienced over that area during the 1992 Cairo Earthquake [12], RE is employed on the edge of the pyramid.

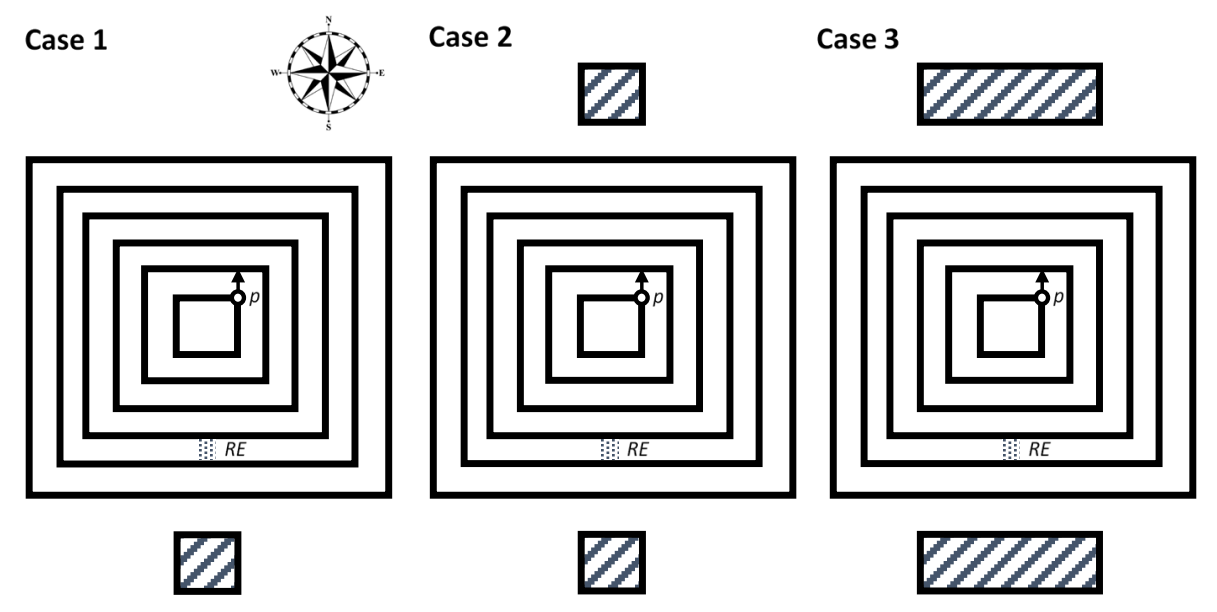

Figure 8. Top view of ViBa configurations for Case 1-2-3
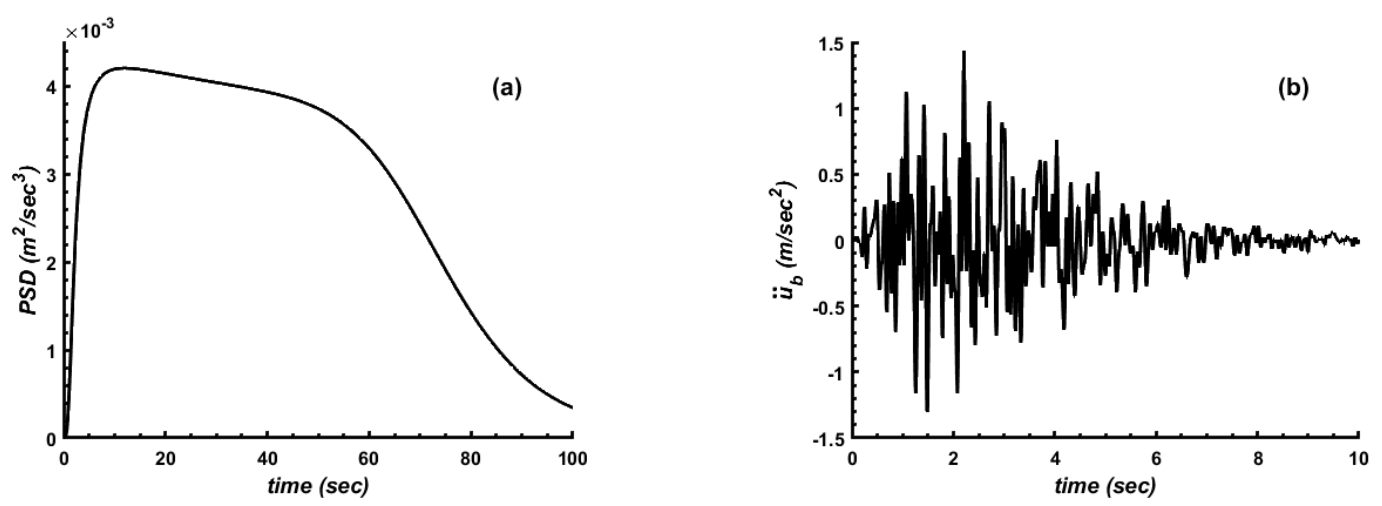

Figure 9. (a) the unilateral PSD function at the bedrock underneath the Zoser Pyramid, (b) a sample of the acceleration time series obtained by the spectral representation method 
The unilateral PSD function (Figure 9a ) at the bedrock, $\operatorname{PSD}(\omega)$, determined using the Boore method [11] and the relevant seismological parameters determined in [12], is used in Eq. (4) of the proposed approach to perform the optimal design of the ViBa. Furthermore, the same PSD function is also used to generate 30 realizations of the time-history acceleration functions using the spectral representation method; these realizations are used to calculate the statistics of the seismic response of the Pyramid before and after being protected by the ViBa technology. A random sample is shown in Figure 9b for illustration purposes.

For each case, the proposed 4-step approach is performed. Results of the steady-state analyses of step i), $U_{p}^{(1)}(\omega)$, are shown in Figure 10a, while results from step ii), $U_{p}^{(2)}(\omega)$, are shown in Figure 10b. Also, in step ii) the soil-foundation impedance, $K_{h}(\omega)$, of Eq. (1) can be computed. In this paper, the static values, $K_{h}(0)=4320 \mathrm{GN} / \mathrm{m}($ Case $1-2)$ and $K_{h}(0)=8077$ $\mathrm{GN} / \mathrm{m}$ (Case 3) are used.
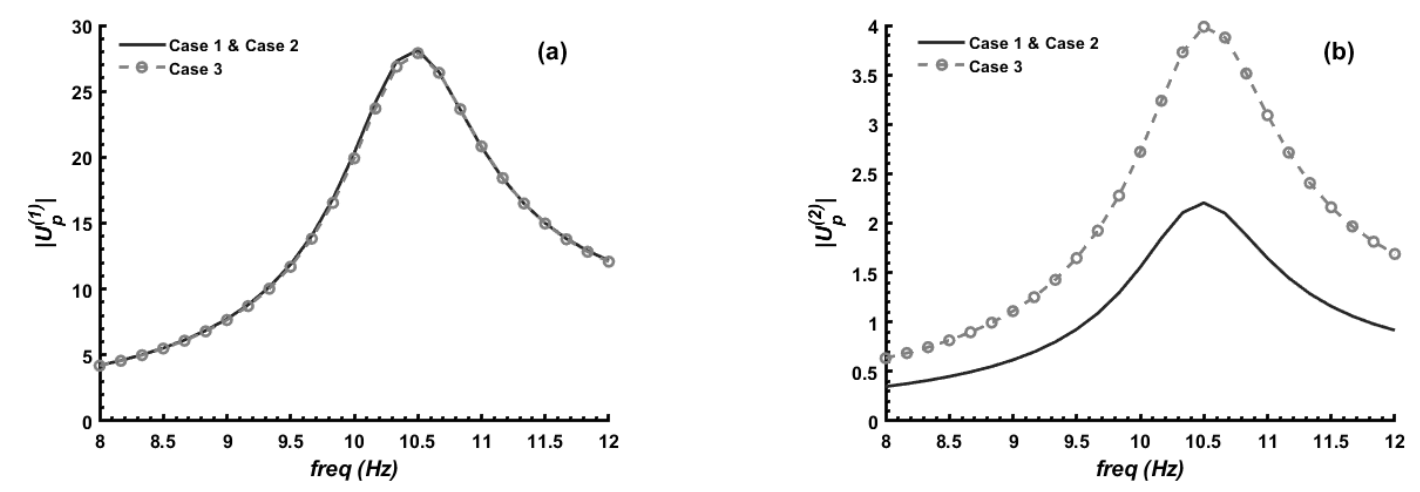

Figure 10. Results of the steady-state analysis in terms of a) $\left|U_{p}^{(1)}\right|$ and b) $\left|U_{p}^{(2)}\right|$ for Case 1-2-3

The optimization problem of Eq. (7) is performed for the 3 cases by considering the total mass of the ViBa devices, $M_{V}$, equal to $80 \%$ of the Pyramid mass, and by fixing the loss factor, $\eta_{V}$, to 0.1 . To minimize the computational effort, a limited range of frequencies from 8 to 12 $\mathrm{Hz}$, hence, around the first natural frequency of the Pyramid, is considered. Optimal parameters computed by using the proposed approach are reported in Table 2.

\begin{tabular}{|c|c|c|c|c|c|c|}
\hline \multirow[b]{2}{*}{ Case } & \multicolumn{3}{|c|}{ ViBa 1} & \multicolumn{3}{|c|}{ ViBa 2} \\
\hline & $M_{V} / M_{p}$ & $\begin{array}{c}K_{V} \\
\mathrm{GN} / \mathrm{m}\end{array}$ & $\eta_{n, V}$ & $M_{V} / M_{p}$ & $\begin{array}{c}K_{V} \\
\mathrm{GN} / \mathrm{m}\end{array}$ & $\eta_{n, V}$ \\
\hline 1 & 0.8 & 7190.6 & 0.1 & - & - & - \\
\hline 2 & 0.4 & 2394 & 0.1 & 0.4 & 1696 & 0.1 \\
\hline 3 & 0.4 & 1883.4 & 0.1 & 0.3 & 1464.2 & 0.1 \\
\hline
\end{tabular}

Table 2. Optimal design of the ViBa for Case 1-2-3.

Figure 11 shows the results of steady-state analyses in terms of power spectral density functions, $G(\omega)=G(\omega, \hat{\alpha})$ for Case 1-2-3, where $\hat{\alpha}$ is the optimal design vector listing the parameters of Table 2. Results, obtained from the full FEM and ROM, are also depicted for a visual comparison. The reduced-order model with just 2 degrees of freedom captures satisfactorily the response of the full ViBa-Soil-Pyramid system. Furthermore, a comparison with the existing scenario without $\mathrm{ViBa}$ is reported with a grey dashed line. It can be seen that the ViBa technology greatly modifies the Pyramid's response by decreasing the total energy experienced by the Pyramid. 


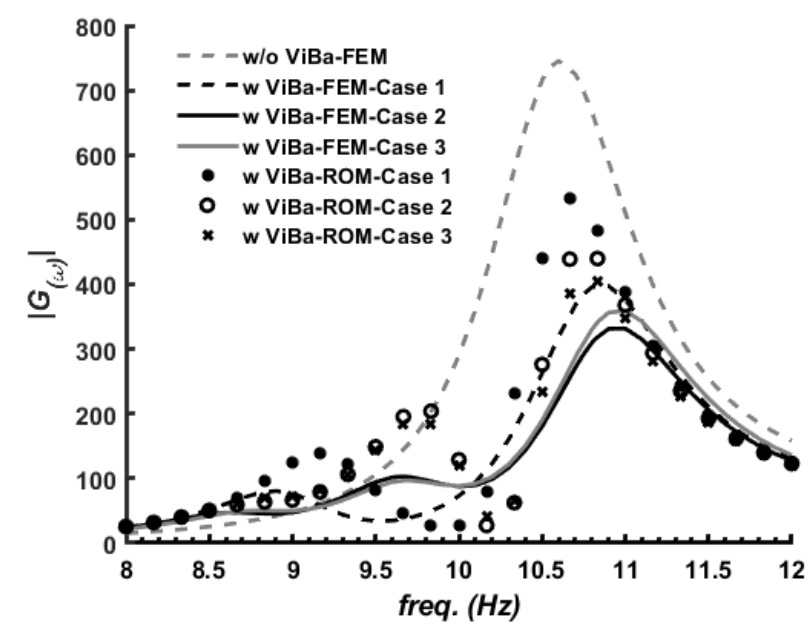

Figure 11. Steady-state response of the pyramid for different cases.
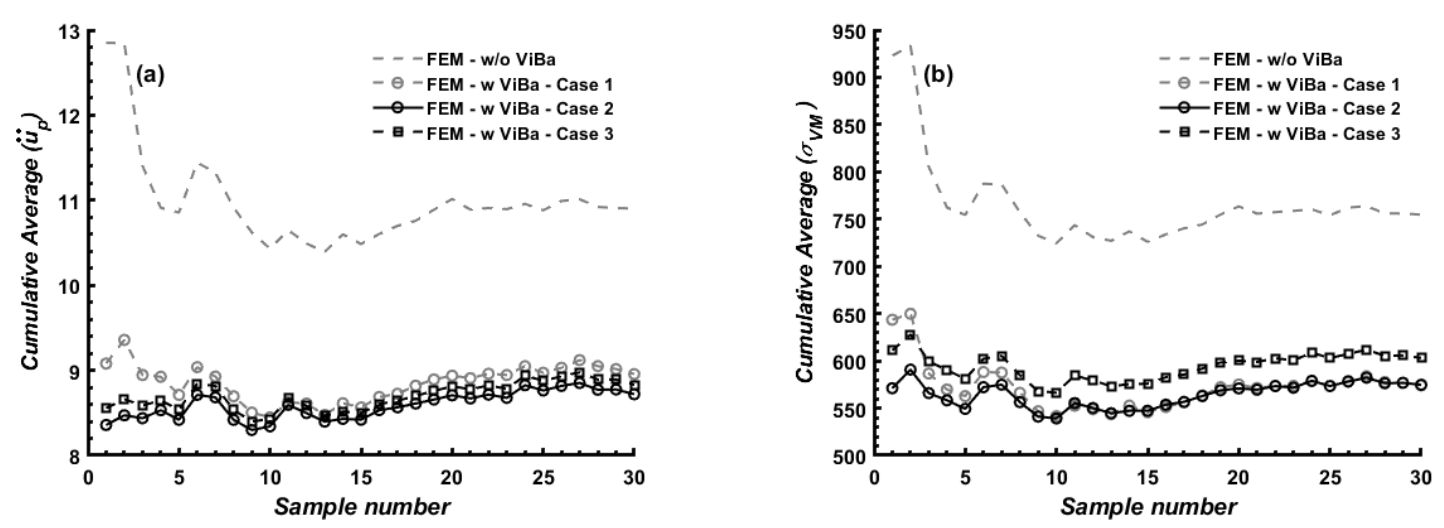

Figure 12. Cumulative average of acceleration recorded at the top of the pyramid (a) and Von Mises Stress (b) recorded at the specific element in respect of the number of realizations.

To assess the effects of the mitigation induced by the ViBa technology on the seismic response of the Pyramid, a modal time-history Monte Carlo Simulation (MCS) is performed by using the $n=30$ generated realizations. The three Cases are compared to the existing scenario of the Zoser Pyramid with no seismic protection. Figure 12 shows the convergence of the results in terms of average acceleration and Von Mises stress at the control point and reference element indicated in Figure 8.

In Figure 13, the mitigating effect of the ViBa technology against the existing scenario without the protection of the ViBa, is shown as acceleration and Von Mises time history functions for Case 1-2-3 for a randomly generated sample. 

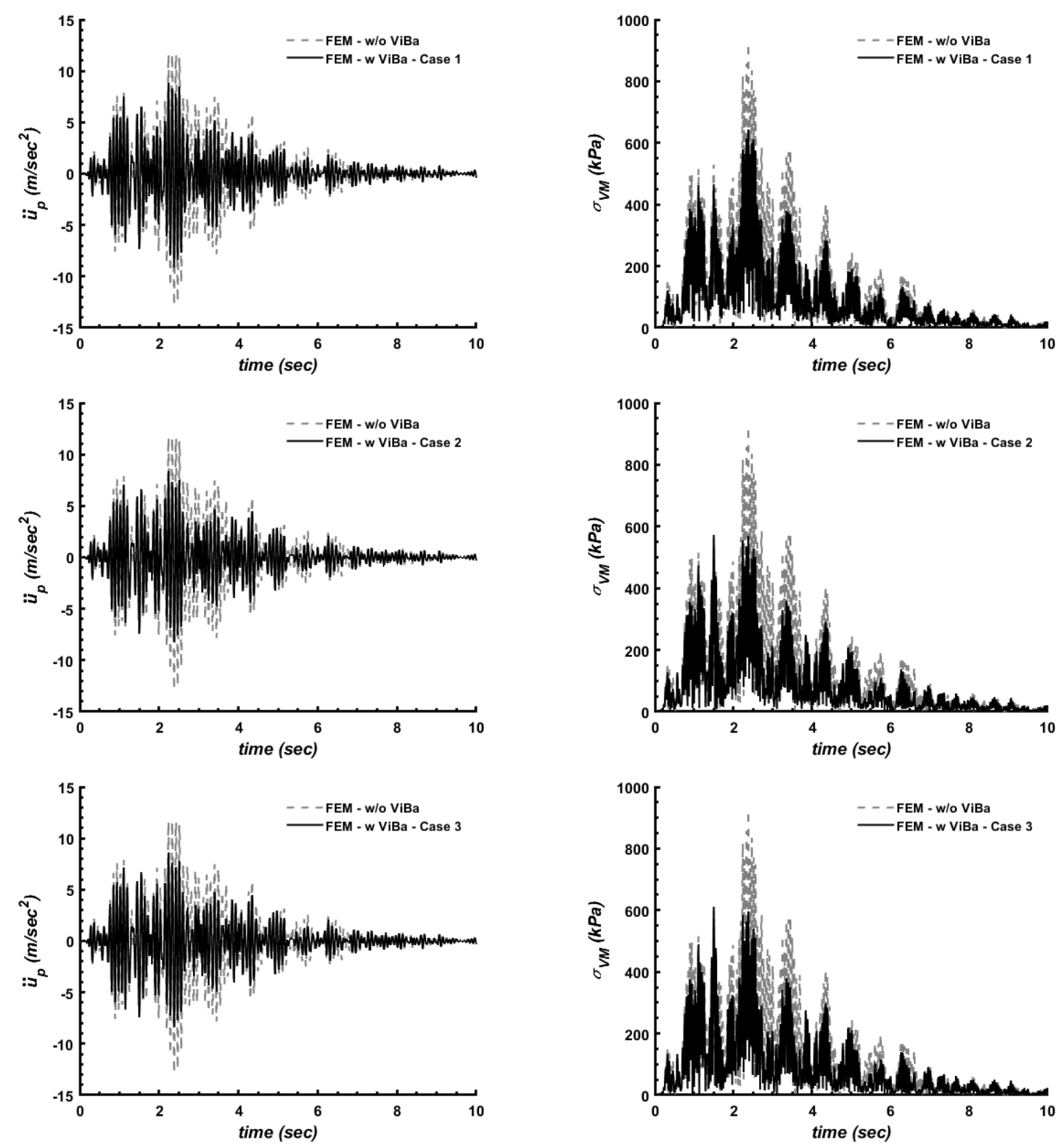

Figure 13. Acceleration time series and Von Mises Stress $\left(\sigma_{V M}\right)$ time series for the top of the pyramid and specified element of the pyramid (RE), respectively, for different cases.

The mitigation of the seismic response is evaluated through a reduction factor in terms of acceleration defined as:

$$
R_{\ddot{u}_{p}}^{j}=\frac{\left|\max \left(\left|\ddot{u}_{p}^{j}(t)\right|\right)-\max \left(\left|\ddot{u}_{p, V i B a}^{j}(t)\right|\right)\right|}{\max \left(\left|\ddot{u}_{p}^{j}(t)\right|\right)} \times 100
$$

where $\ddot{u}_{p}^{j}(t)$ and $\ddot{u}_{p, V i B a}^{j}(t)$ are the accelerations computed at the reference point of the Pyramid induced by the $\mathrm{j}$-th realization for the full FEM model, before and after being protected by the ViBa technology, respectively. Therefore, the mean value is expressed as: 


$$
\bar{R}_{\ddot{u}_{p}}^{F E M}=\frac{1}{n} \sum_{j=1}^{n} R_{\ddot{u}_{p}}^{j}
$$

A further expression of the reduction factor in terms of Von Mises stresses is defined as follows:

$$
R_{\sigma}^{j}=\frac{\left|\max \left(\left|\sigma_{V M}^{j}(t)\right|\right)-\max \left(\left|\sigma_{V M, V i B a}^{j}(t)\right|\right)\right|}{\max \left(\left|\sigma_{V M}^{j}(t)\right|\right)} \times 100
$$

where $\sigma_{V M}^{j}(t)$ and $\sigma_{V M, V i B a}^{j}(t)$ are the Von Mises stresses computed at the horizontally centralsecond row-edge element of the pyramid induced by the $\mathrm{j}$-th realization for the full FEM model, before and after being protected by the ViBa technology, respectively. Accordingly, the mean value is determined as:

$$
\bar{R}_{\sigma}^{F E M}=\frac{1}{n} \sum_{j=1}^{n} R_{\sigma}^{j}
$$

The mitigation induced by the ViBa on the seismic response of the Pyramid is shown in Figure $14 \mathrm{a}-\mathrm{b}$ for both coefficients determined by Eq. (8) and (10). It is worth noting that the effect of the $\mathrm{ViBa}$ is beneficial for most of the events (only a few cases have been observed in which the $\mathrm{ViBa}$ was ineffective or slightly detrimental $<2 \%$ ) with a maximum reduction equal to $35 \%$ on the peak acceleration and $39 \%$ on the Von Mises stresses.
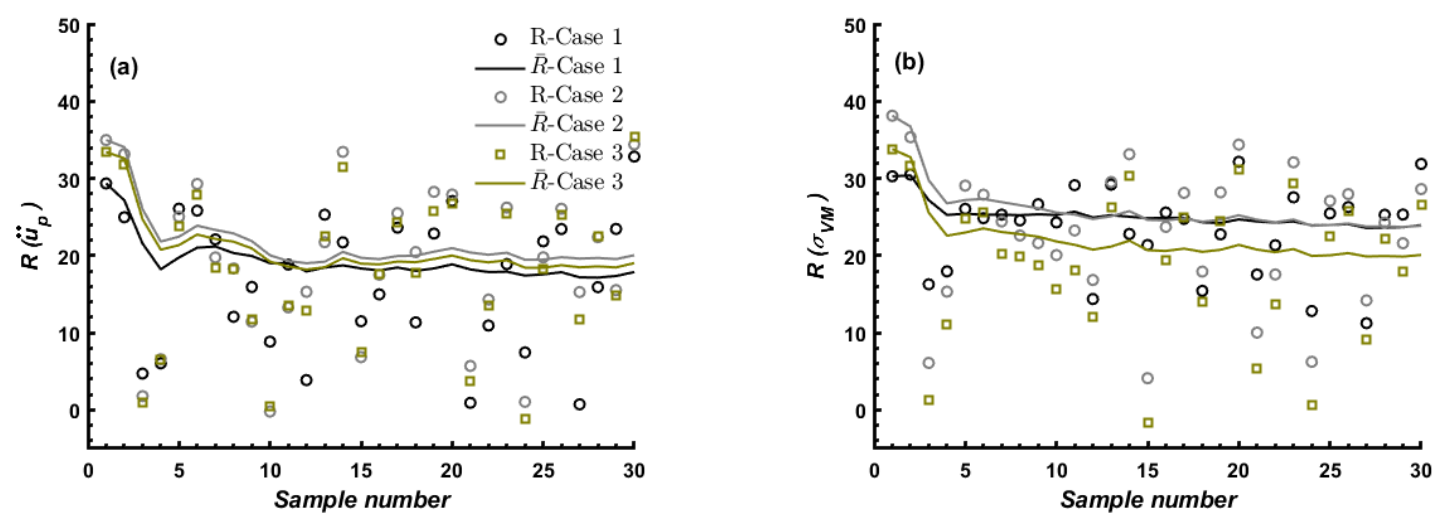

Figure 14. The mitigation provided by ViBa on the seismic response of the Pyramid; (a) mitigation on maximum acceleration, (b) mitigation on maximum Von Mises Stress.

A summary of the results is reported in Table 3 in which the computed averages of Eq. (9) and (11) from the MCS are compared with the target reduction obtained by the ROM through Eq. (7). Therefore, good reductions around $19 \%$ on the peak acceleration and about $23 \%$ on the Von Mises stress are observed for all three Cases A slight improvement of the reduction of the peak acceleration is obtained for Case 3 (about 25\%) whilst a higher reduction of the stresses on the reference element is obtained for Case 1-2 (about 24\%). Remarkably, the three configurations used in this paper do not significantly affect the outcome once the same mass ratio is used, hence, more ViBas with smaller individual masses might be used to efficiently mitigate the seismic response of the Pyramid. 


\begin{tabular}{|c|c|c|c|c|l|}
\hline Case & $R_{\ddot{u}_{p}}^{R O M}(\%)$ & $\bar{R}_{\ddot{u}_{p}}^{F E M}(\%)$ & $\begin{array}{l}\text { Rel. err } \\
(\%)\end{array}$ & $\bar{R}_{\sigma}^{F E M}(\%)$ & $\begin{array}{l}\text { Rel. err } \\
(\%)\end{array}$ \\
\hline 1 & 20.95 & 17.86 & 14.75 & 23.94 & $-14.27 \%$ \\
2 & 23.219 & 20.04 & 13.69 & 23.86 & $-2.76 \%$ \\
3 & 24.46 & 19.02 & 22.24 & 20.08 & $17.91 \%$ \\
\hline
\end{tabular}

Table 3. Reduction of the Pyramid response in terms of max acceleration and stresses

It is worth mentioning that the ViBa technology affects the whole behaviour of the Pyramid and not just the investigated control point and reference element. Therefore, the complete contour map of the Von Mises stresses is also shown for a specific instant, $t=2.38 \mathrm{~s}$, in which the Pyramid experienced the maximum stress at the reference element. Figure 15 shows the distribution of the stresses for the existing situation subjected to the first realization; the maximum stresses occur in the same region where the Pyramid has been largely damaged during the 1992 Cairo earthquake event.

Figure 16 shows the impact of the excavation on the stresses of the Pyramid for Case 1 and 3 for the same realization used for Figure 15. In both cases, the changes in the distribution of the stresses on the Pyramid is minimal, indicating that the excavation on its own cannot reduce the seismic body waves as opposed to the effect of trenches on surface waves (see e.g. [17]).

(a)
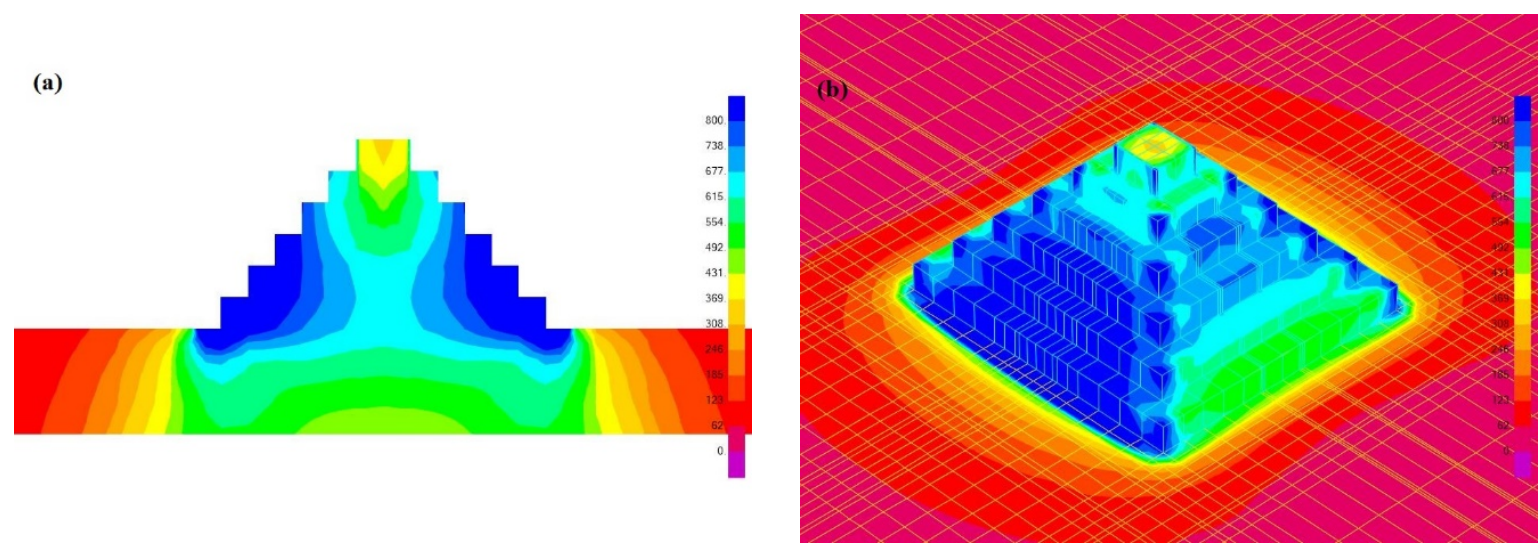

Figure 15. Instantaneous Von Mises stress contour map of a) the central cross-section and, b) the 3D soil-pyramid system for the first generated earthquake.
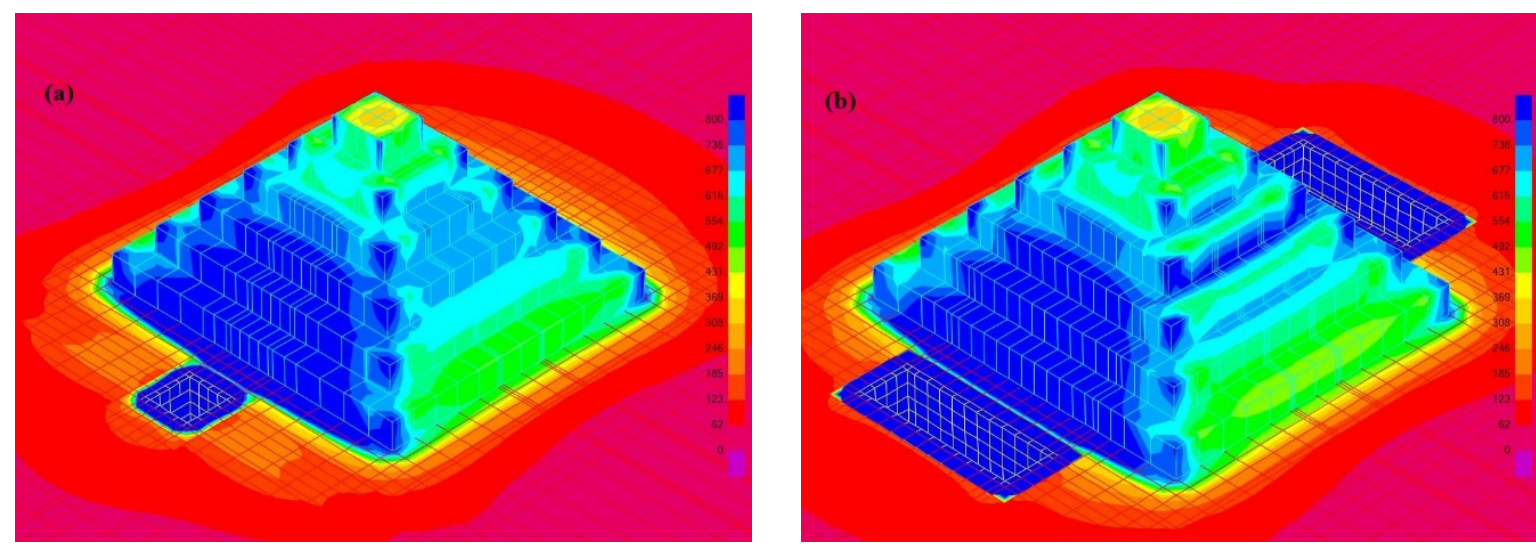

Figure 16. Effect of excavation on the response of the pyramid, (a) small excavation on one side of the pyramid, (b) large excavation on both sides of the pyramid. 
Figures 17-18-19 show the response of the Pyramid protected by the ViBa device. As it can be seen, the Von Mises stresses are reduced over a large volume of the Pyramid. Particularly in Case 2, the ViBas have a narrower but greater influence over the central area whilst the large ViBas on Case 3 act in a more uniform fashion.

(a)
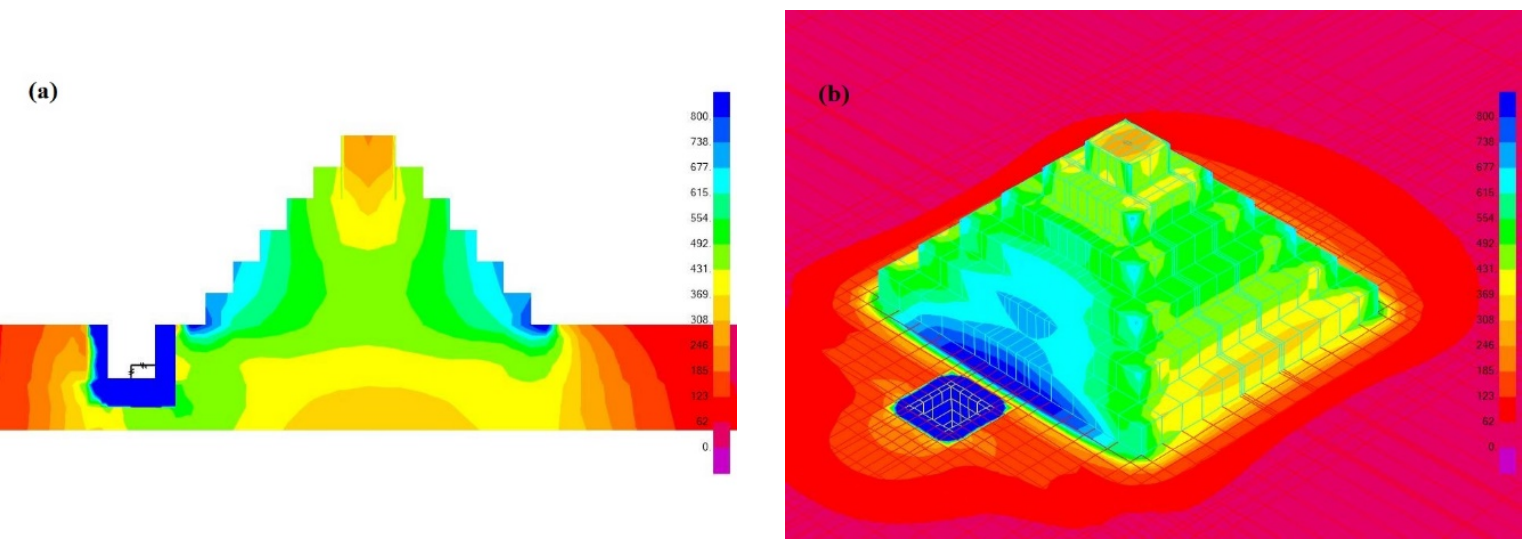

Figure 17. Maximum Von Mises stress contour map in the central cross-section and 3D for case 1 for the first generated earthquake.

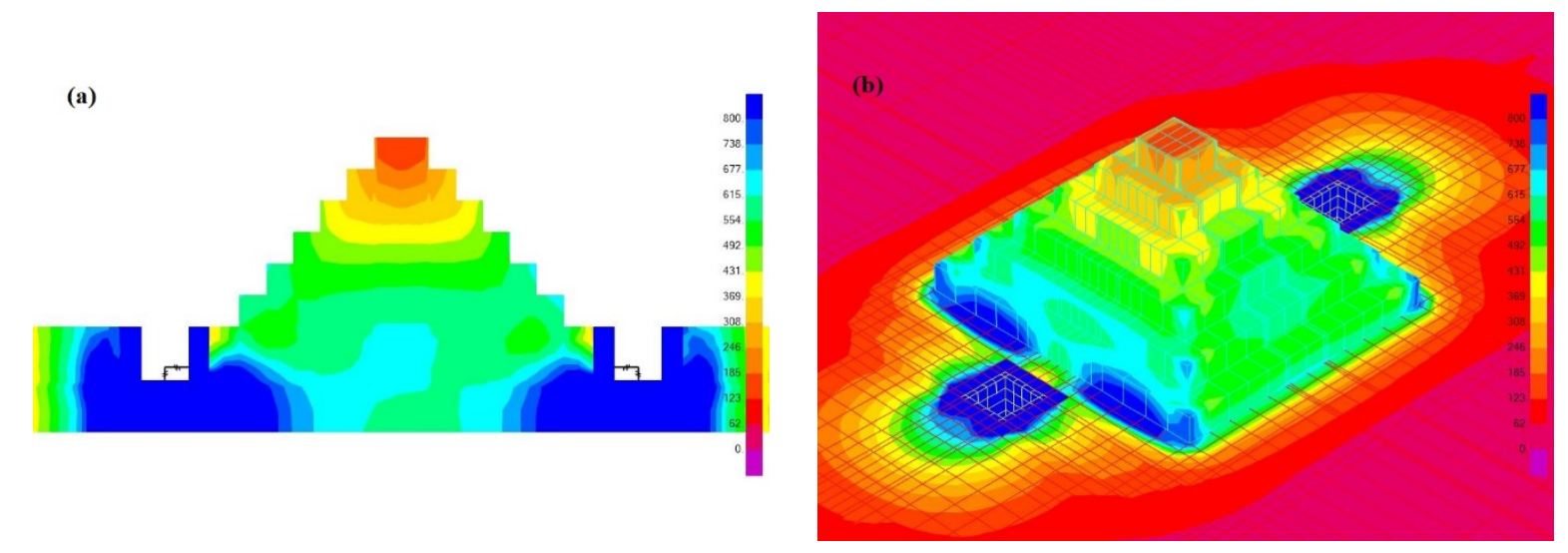

Figure 18. Maximum Von Mises stress contour map in the central cross-section and 3D for case 2 for the first generated earthquake.

(a)
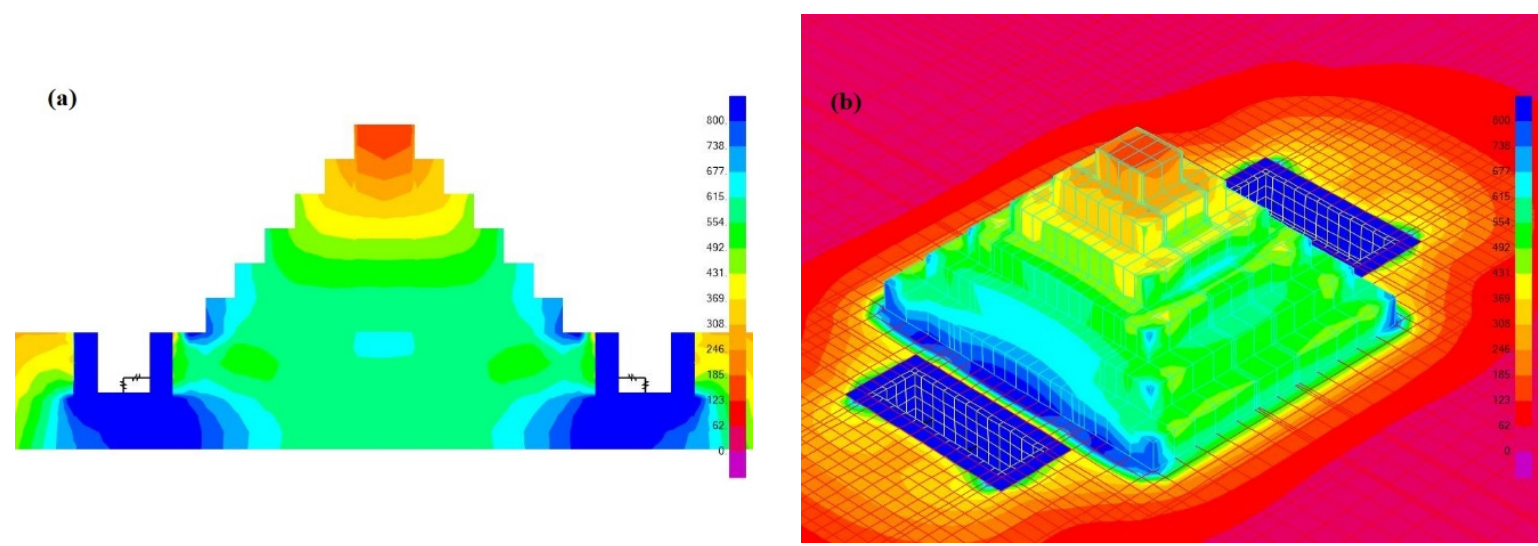

Figure 19. Maximum Von Mises stress contour map in the central cross-section and 3D for case 3 for the first generated earthquake. 


\section{CONCLUSIONS}

In this paper, the innovative non-invasive vibration control technology called Vibrating Barrier has been applied to reduce the seismic response of the Step Pyramid of Zoser. The Pyramid has been severely damaged during the 1992 Cairo Earthquake and to guarantee its preservation, the seismic risk should be mitigated. To reduce the computational complexity of the model during the design stage of the ViBa parameters, a 4-step approach has been proposed to derive a Reduce-Order Model by adopting a sub-structuring technique. Only 2-DOFs capturing the $\mathrm{ViBa}$ translations of the internal device and its foundation is required. The result of the inertial analysis is then related to the seismic behaviour of the Pyramid through non-dimensional transfer functions obtained from steady-state analyses on the full FEM Soil-Pyramid system. Because the transfer functions are computed only once, this proposed approach allows performing an optimization analysis on the several parameters of the ViBa through a very fast and robust procedure. By considering a spectral representation of the 1992 Cairo earthquake, the results in terms of peak acceleration and stresses on particular control points have shown a significant reduction of the seismic response of the Pyramid when the ViBa device is used. An average reduction ranging from $20 \%$ to $24 \%$ has been observed on the Von Mises stresses with maximum mitigation of up to $39 \%$. Remarkably, reductions have been observed in those areas where the damages have been observed. Parametric studies changing the configuration of the ViBa with one or two box-foundations and small and large individual masses were also conducted to assess their impact on efficiency. Whilst results have been obtained with a large overall mass of $80 \%$ of the Pyramid mass, the parametric analysis shows that several ViBa devices can be used with smaller masses or by improving its technology through inerters as done in [16]. Therefore, because of the impossibility to use conventional seismic control solutions without affecting its cultural heritage, the $\mathrm{ViBa}$ technology can be seen as a viable solution to guarantee the preservation of the ancient monuments as the Zoser pyramid.

\section{ACKNOWLEDGEMENTS}

This research was supported by the AHRC Grant AH/R007934/1 Preserving Egypt's Cultural Heritage from Earthquake Damage through Vibrating Barriers (ViBa).

\section{REFERENCES}

[1] E.D. Johnson, The Need for Seismic Analysis and Planning as Part of Ongoing Archaeological Site Management and Conservation: A Case Study of the Necropolis of Saqqara, J. Am. Res. Cent. Egypt. 36 (1999) 135-147. https://doi.org/https://doi.org/10.2307/40000207.

[2] P. Cacciola, A. Tombari, Vibrating barrier: a novel device for the passive control of structures under ground motion, Proc. R. Soc. London A Math. Phys. Eng. Sci. 471 (2015). https://doi.org/https://doi.org/10.1098/rspa.2015.0075.

[3] G. Warburton, J. Richardson, J. Webster, Forced Vibrations of Two Masses on an Elastic Half Space, J. Appl. Mech. ASME. 38 (1971) 148-156. https://doi.org/10.1115/1.3408735.

[4] J. Luco, L. Contesse, Dynamic structure-soil-structure interaction., Bull. Seismol. Soc. Am. 63 (1973) 1289-1303.

[5] P. Cacciola, M.G. Espinosa, A. Tombari, Vibration control of piled-structures through structure-soil-structure-interaction, Soil Dyn. Earthq. Eng. 77 (2015) 47-57.

[6] A. Tombari, I. Zentner, P. Cacciola, Sensitivity of the stochastic response of structures 
coupled with vibrating barriers, Probabilistic Eng. Mech. 44 (2016) 183-193.

[7] P. Cacciola, N. Banjanac, A. Tombari, Vibration Control of an existing building through the Vibrating Barrier, in: Procedia Eng., 2017. https://doi.org/10.1016/j.proeng.2017.09.065.

[8] M. Andreozzi, A. Tombari, A. Lampropoulos, P. Cacciola, Seismic protection of the Messina Cathedral Bell Tower through Vibrating Barriers, in: SECED 2019 Earthq. Risk Eng. Towar. a Resilient World, Greenwich, UK, 2019.

[9] J.D. Coronado, R. Lomurno, A. Tombari, P. Cacciola, Improving Urban Seismic Resilience through Vibrating Barriers, in: ICOSSAR, Vienna, Austria, 2017.

[10] A. Tombari, M. Garcia Espinosa, N.A. Alexander, P. Cacciola, Vibration control of a cluster of buildings through the Vibrating Barrier, Mech. Syst. Signal Process. (2018). https://doi.org/10.1016/j.ymssp.2017.08.034.

[11] D. Boore, Simulation of Ground Motion Using the Stochastic Method, Pure Appl. Geophys. 160 (2003) 635-676.

[12] A.E. Khalil, H.E. Abdel Hafiez, M. Girgis, M.A. Taha, Earthquake ground motion simulation at Zoser pyramid using the stochastic method: A step toward the preservation of an ancient Egyptian heritage, NRIAG J. Astron. Geophys. 6 (2017) 52-59. https://doi.org/https://doi.org/10.1016/j.nrjag.2016.11.003.

[13] M. Metwaly, A.G. Green, H. Horstmeyer, H. Maurer, A.M. Abbas, A.R.G. Hassaneen, Combined seismic tomographic and ultra-shallow seismic reflection study of an early dynastic mastaba, Saqqara, Egypt, Archaeol. Prospect. 12 (2005) 245-256. https://doi.org/10.1002/arp.261.

[14] G.M. El-Qady, F.A. Monteiro Santos, A.G. Hassaneen, L. Trindade, 3D inversion of VES data from the Saqqara archaeological area, Egypt: a case study, Near Surf. Geophys. 3 (2005) 227-233. https://doi.org/10.3997/1873-0604.2005018.

[15] J. Lysmer, F. Ostandan, C.C. Chin, SASSI 2000-A System for Analysis of SoilStructure Interaction-User's Manual, Berkeley, California: University of California., 1999.

[16] P. Cacciola, A. Tombari, A. Giaralis, An inerter-equipped vibrating barrier for noninvasive motion control of seismically excited structures, Struct. Control Heal. Monit. 27 (2019) 1-21.

[17] E. Çelebi, S. Firat, I. Çankaya, The effectiveness of wave barriers on the dynamic stiffness coefficients of foundations using boundary element method, Appl. Math. Comput. 180 (2006) 683-699. 